\title{
Disentangling the dynamic core: a research program for a neurodynamics at the large-scale
}

\author{
MICHEL LE VAN QUYEN \\ Laboratoire de Neurosciences Cognitives et Imagerie Cérébrale, Paris, France
}

\begin{abstract}
My purpose in this paper is to sketch a research direction based on Francisco Varela's pioneering work in neurodynamics (see also Rudrauf et al. 2003, in this issue). Very early on he argued that the internal coherence of every mental-cognitive state lies in the global self-organization of the brain activities at the large-scale, constituting a fundamental pole of integration called here a "dynamic core". Recent neuroimaging evidence appears to broadly support this hypothesis and suggests that a global brain dynamics emerges at the large scale level from the cooperative interactions among widely distributed neuronal populations. Despite a growing body of evidence supporting this view, our understanding of these large-scale brain processes remains hampered by the lack of a theoretical language for expressing these complex behaviors in dynamical terms. In this paper, I propose a rough cartography of a comprehensive approach that offers a conceptual and mathematical framework to analyze spatio-temporal large-scale brain phenomena. I emphasize how these nonlinear methods can be applied, what property might be inferred from neuronal signals, and where one might productively proceed for the future. This paper is dedicated, with respect and affection, to the memory of Francisco Varela.
\end{abstract}

Key terms: nonlinear dynamics, phase synchronization, chaos, EEG, non-gaussian statistics

\section{INTRODUCTION: THE CORE HYPOTHESIS}

Although much is known about the structure and functional attributes of the brain, a comprehensive understanding of its largescale dynamical properties remains unknown. At this macroscopic level, a prevalent feature of the brain activity is the presence of metastable spatio-temporal patterns of neural activity distributed over many distinct brain regions. The existence of large-scale cortical dynamics is confirmed by numerous functional brain mapping studies, employing electro- and magneto-encephalography (EEG-MEG), functional Magnetic Resonance Imaging (fMRI) or Positron Emission Tomography (PET). As suggested by these observations, a key property of this large-scale dynamics is its extraordinary degree of coordination. The different parts of the brain cooperate through their rich network of connections to produce a unified whole, typically including multiple modalities and submodalities, which cannot be subdivided into independent components without a loss in its meaning. A major question of modern neuroscience is how the (non-linear) coupling between neuronal components gives rise to a wide variety of complex, coordinated behaviors (Damasio, 1990; Kelso 1995; Varela, 1995; Singer and Gray, 1995; Tonini and Edelman, 1998; Friston, 2000).

This question was put at the center stage of neurobiological research starting with studies showing that neuronal processes should be considered as non-local cooperative integrations across spatially distributed local regions. Prominent among such studies is the research of W. Freeman on mass action in the nervous system using macro-potential in awake animals (Freeman, 1975). Very early, Francisco Varela followed up Freeman's pioneering insights (Varela, 1979) and worked on these 
questions until his death (see Rudrauf et al. 2003, in this issue). In this context, he formulated perhaps the most audacious and comprehensive approach to the explication of global integrative processes (1995): For every cognitive act, there is a singular and specific large cell assembly that underlies its emergence and operation. In his view, the generation of a mental-cognitive state corresponds to the transient constitution of one dominant assembly which incorporates or discards into its components multiple neuronal activities distributed over both cortical and subcortical regions (Fig. 1-A). More recently, Tononi \& Edelman (1998) termed this process as the "dynamic core," in order to emphasize both its integration and its constantly changing activity patterns. According to Varela's working
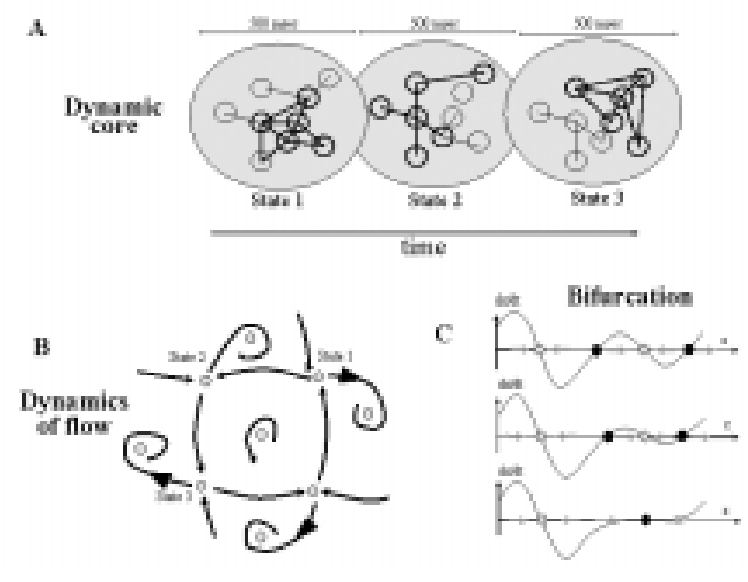

Figure 1: A: Diagram depicting the dynamic core hypothesis. For every mental-cognitive state, a specific large cell assembly is generated, incorporating into its components multiple distributed neuronal activities. B: The dynamics for this emergent behavior can be described geometrically, in terms of positions, regions and trajectories in a state space. C: Changes in dynamics occur through bifurcations. As an example, the saddle-node bifurcation is illustrated here (from top to bottom): The temporal evolution of a variable, $x$, is characterized by a dynamical system $d x / d t=f(x)$. In the example shown, there are two attractors (circles in gray) and two repellors (circles in black). As the right-most attracting state becomes less stable (negative slope of rate of change at fixed point approaches zero), the flanking repellors approach, until their collision removes the stable, leaving a single repellor behind. hypothesis, this endogenous dynamics has three main characteristics: First, the dynamic core constantly gives rise to new patterns in succession and thus is dynamically unstable. This is the notion of metastability, which is central to the entire idea of large-scale dynamics presented here since it underscores that the global activity enters into different states of coordination of its constituent areas without becoming trapped in any one particular state. Second, the dynamic core provides a reference pole that dynamically modulates the microscopic cellular interactions and locally constrains their behaviors. A reciprocal interplay between a relative local autonomy of the elementary neuronal components and the global web of interdependences creates a wide variety of complex, global states that are able to carry out different behaviors. Finally, the dynamic core must emerge very quickly, in a time frame of 100 to 300 msec. Such rapid integration can be achieved through the strong cortico-cortical and cortico-thalamic projections generating a coherent neuronal process through ongoing re-entrant, recursive, and highly parallel interactions among widely distributed brain areas. These three main characteristics allow the brain as a whole to switch rapidly between different functions through the reorganization of its component areas into different coordinated networks.

In addition to experimental works, the plausibility of this view has been demonstrated by large-scale simulations (65,000 integrate-and-fire neurons and 5 million connections) of thalamocortical circuits in the brain (Lumer et al, 1997). Clearly, the resulting network reacts to perturbations in a non-linear fashion. For stimulus input intensities below a certain threshold (defined by the parameters of the model), the system only exhibits lowintensity, background activity. As the intensity of the input rises beyond the threshold, the dynamics of the system manifests a phase transition: the activity of the system changes suddenly to a stable oscillatory mode with a frequency of about 20-60 Hz and a large amplitude (compared to background activity). Dynamically, it has been shown that these transiently stable 
collective oscillations emerge as a consequence of the re-entrant interactions in the various simulated brain areas in response to the stimulus input.

Despite a growing body of evidence supporting this view, our understanding of these large-scale brain processes remains hampered by the lack of a theoretical language for expressing these complex behaviors in dynamical terms. In the course of the last decade, the recent emergence of a general theoretical framework that explicitly considers nonlinear nonequilibrum, open and strongly coupled systems have provided a new opportunity for neuroscience to pursue the analysis of brain self-organization. Here the question I wish to address is to what extent these descriptions can improve our understanding of the large-scale brain dynamics. In particular, I sketch a rough cartography of different nonlinear techniques which may be of relevance for this description. I have not attempted an exhaustive review of these methods. In contrast, the main purpose of this paper is, as the title indicates, to outline a research direction based on Francisco Varela's neurodynamics. In particular, I address these issues from a pragmatic point of view, based on my own work, and the emphasis is explicitly put where one might productively proceed for the future.

\section{THEORETICAL FOUNDATIONS}

In physics, what is usually referred to as self-organization is the spontaneous formation of well organized structures, patterns, or behaviors, from random initial conditions. Typically, these systems possess a large number of elements or variables interacting in a complex way, and thus have very large state spaces. However, when started with some initial conditions, they tend to converge to small areas of this space which can be interpreted as a form of emergent eigenbehavior. The earliest example for this behavior in a simple physical system is the convection cells (Bénard instability) in a horizontal water layer with a thermal gradient. This example shows that when the thermal gradient has reached a critical value, the initially uncorrelated, random motions of billions of molecules synchronize spontaneously without any external control, leading to a spontaneous emergence of long-range correlated macroscopic structures (Chandrasekhar, 1961).

During the early 1970s, the growing need for a better understanding of these types of cooperative behaviors led to a general theoretical description based on a branch of mathematics called dynamic system theory (Nicolis and Prigogine, 1977). In a nutshell, the traditional starting point of all these descriptions consists of conceptualizing the dynamics geometrically, in terms of positions, regions and trajectories in a space of possible states. Collectively, they constitute what is known as the system flow. The shape of the flow is determined by the system's intrinsic dynamics - the force that pushes the system state in one direction or another, depending on where the current state is located. They can be thought of as constituting a kind of landscape over which the behavior of the system moves. The global shape of dynamical landscape is determined by the so-called order parameters. For autonomous dynamical systems, these global parameters are solely created by the intrinsic interactions among the individual parts of the system (see section 8 for an example). They in turn govern the behavior of the individual parts and this is sometimes referred to as a circular causality. The dynamics of these order parameters (by definition, this dynamics is slower than that of the system) characterizes how the dynamical landscape is formed and evolves in time. In fact, a change in the order parameter affects every possible path (the entire flow) and can give rise to a qualitatively new dynamical landscape. Such changes give rise to bifurcations, also called instabilities. In a quite general way, bifurcations in the system demarcate different stable behaviors, thus enabling identification of the parameter domains on which this stability occurs. 


\subsection{What does it mean for neurosciences?}

In the early 1950s, Ashby made the startling proposal that all the brain phenomena might be accounted for with dynamical system models (1952), but little work directly followed from his speculation due to a lack of appropriate mathematical methods and computational tools to implement practical models. More recently, the development of connectionist models since the 1980s has certainly helped to move the neurosciences in the direction of dynamical thinking (Hopfield,1982), but a thoroughly dynamical approach is the study of phenomena occurring in continuous time something not all connectionist models do. Recently, dynamical descriptions have provided a promising way to model complex motor behaviors and to characterize the relative stability of possible patterns of changes. Among others, Kelso (1995) in his experiment on phase transitions during finger movement, found that these properties can be described and predicted in detail by assuming that a single, continuous, high level, «collective» variable - the relative phase - evolves in a way governed by a suitable form of a simple differential equation (the so-called HKB model). In this model, in-phase and antiphase patterns of coordination were identified as two elementary behaviors, which coexist bi-stably at a low frequency of the rhythmic movement. Anti-phase coordination (syncopation) becomes unstable in a saddle-node bifurcation (Figure 1-C) when movement frequency is increased. The basic insight here is that states that may coexist multi-stably or are linked through bifurcations reflect separate elementary behaviors or neural patterns. Another noteworthy fact about this model is that the used variables are not low-level (e.g., neural firing rates), but rather macroscopic quantities at roughly the level of the behavior itself.

According to this view, a central idea pursued by F. Varela is that the dynamical system descriptions may provide guidance to a new interpretation of the global brain dynamics as a flow (Varela, 1999). Why is this of importance here? First, these descriptions lead us directly to an explicit view of the dynamic core as a trajectory in a state space (Fig. 1B). Because of its intrinsic instability, no stable regions exists in the phase space, but rather ongoing sequences of transient visits of specific weakly attracting places in a complex pattern of motion, modulated by external influences. Second, these descriptions make explicit generic structures of the dynamic core, i.e. characteristics that are observed independently of the particular variation of the context or the components of the network under consideration. The key feature is here given by the intrinsic dynamical landscape that brings the flow into particular trajectories, depending on where the current state is located. As stressed above, this geometry of the state space is a generic property of the dynamics and fundamentally distinct from the particular trajectories that move within it. Finally, dynamic system theory can help us to describe order parameters of the dynamic core, characterizing how its intrinsic dynamics is constituted and unfolds in time, possibly around some instabilities. Again, these global parameters emerge from the intrinsic activity of the nervous system itself and constrain the behavior of the individual parts of the brain (see paragraph 6.2 for an example).

In many ways dynamics has a natural appeal for neurosciences, since it provides ways to conceptualize systems undergoing continual change, ways to characterize the relative stability of possible patterns of change as a function of system parameters, and ways to think about couplings between complex processes such as local neural activities distributed over many distinct brain regions. In a general way, the dynamical system descriptions address the fundamental conflict between stability and instability of brain large-scale dynamics. Stability is the property of a neural state to persist in the face of systematic variation or random perturbation. All observable global brain states possess stability to some extent, as loss of stability leads to change of state. The generation of behavior or of neural patterns of activity is unstable in the sense that a variety of behavioral or neural 
patterns may be assembled from a multitude of sensory, motor, and neural systems in response to the sensory situation, the inner state of the neural or behavioral system, and its recent history. This flexibility may therefore involve pattern change and hence require the release of behavioral or neural states from stability. As introduced in the following, dynamical systems approaches provide methods with which meta-stable states and changes in the number and nature of attractors as sensory information, environmental conditions, or task requirements vary can be identified and characterized.

\section{THE NONLINEAR TECHNIQUES: A CARTOGRAPHY OF THEIR DOMAINS OF RELEVANCE}

From the perspective given by the dynamic system theory, we have a mathematical foundation that offers a characterization of complex behaviors that is far richer than that obtained by classical measures. Recently, the dynamic system theory promoted the development of a huge number of analysis techniques, often referred to as nonlinear time series analysis. Here, it is clear that the mathematical rigor is not always easy to translate to the biological domain, and there is no doubt that applications to the brain processes is still open to considerable progress. Moreover, in contrast to the linear description which can all be cast within the single convenient framework of the Fourier analysis, there are many ways in which the neuronal dynamics may be considered to behave in a nonlinear manner. As previously proposed (Schreiber, 1999), the domain of application of each techniques can be characterized by some hypothesis about 1) the degree of nonlinearity (how nonlinear is the process?) and 2) the degree of stochasticity (how much is the dynamics ruled by noise terms?). Figure 2 shows each domain of application and fundamental hypothesis of the different families described in the text. Note that although these techniques have reached a high degree of maturity and sophistication, their applications to neuroscience are still in their infancy. As our motivation here, a clear physiological interpretation to the output of a particular technique must thus be given and the assumptions underlying a particular technique have to be compatible with the properties of the data.

A large body of evidence has shown that intrinsically different processes of the brain's dynamics tend to dominate in distinctive, characteristic domains of scale. Concerning spatial levels, for example, although the microscopic neuronal interactions are strongly nonlinear, and probably chaotic, recent model of EEG generation have also suggested that the macroscopic activity exhibits linear dynamics (Wright and Liley, 1996). Therefore, events at cellular and global scale may be studied to some degree in isolation with different types of dynamical description. On the other hand, a few different scales of duration can be distinguished in brain processes, each expressing their own dynamics. Varela (1999) identified three ranges of complexity in terms of the dynamical properties: the scale of the intrinsic cellular rhythms from 10 to $100 \mathrm{msec}(1 / 10 \mathrm{scale})$, the scale of large scale integration on the order of 100-

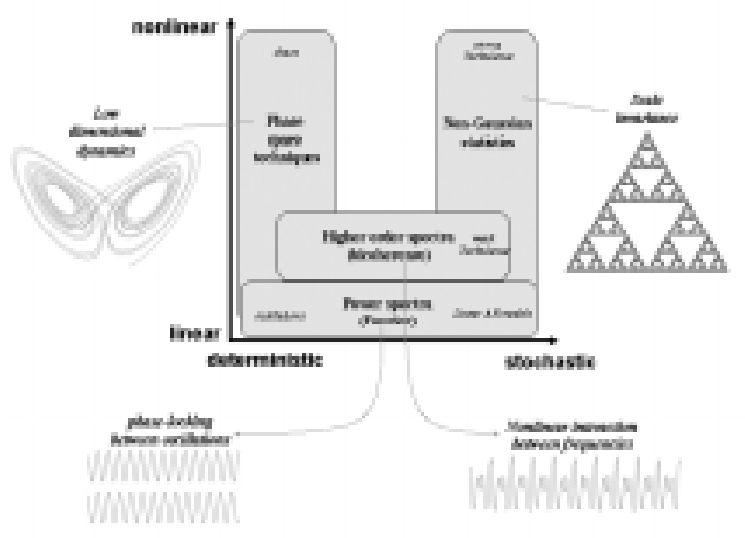

Figure 2: A current cartography of dynamical analysis techniques. The domain of application of each technique can be characterized by some hypothesis about the degree of "stochasticity" and "nonlinearity." The fundamental hypothesis of each families are here schematically depicted by an illustration and are described in the text. Inspired by a similar representation by Schreiber (1999). 
$300 \mathrm{msec}$ (1 scale), and the scale of longterm processes over a few seconds (10 scale). Clearly, these different temporal scales are blended together in a unified dynamics. Nevertheless, as a basic assumption throughout this paper, we assume that several descriptions are needed to investigate these distinctive, characteristic domains of observation (Fig. 7B): At finer time scales (between 1/10 and 1), coupled limit-cycle oscillations are dominant and are best described by spectral representations (section 4 and 5) or weak departure from linear behaviors (section 5 ). Between scale 1 and 10, the absence of clear periodic behavior suggests that local neuronal interactions may rapidly lose information about their initial conditions and phase space techniques (section 6) are expected to be more relevant (Pezard et al., 1994). At scale 10, a disorganized complexity occurs because a system has a large number of significant components that exhibit a high degree of random behavior, and high order statistics (Linkenkaer et al., 2001, section 7) are thus more appropriate for providing dynamical information about these stochastic processes.

\section{CONSTITUTION OF THE DYNAMIC CORE: PHASE SYNCHRONIZATION AND GRAPH DYNAMICS}

At fast time scales (1/10), the formation of the dynamic core demands the coordination of many different regions through a kind of temporal resonance or "glue." Neural synchrony is an important candidate for this integration, mediated by neuronal groups that oscillate in specific bands and enter into precise phase-locking over a limited period of time (Varela et al., 2001). This role of synchronization of neuronal discharges, although not a new idea, has been greatly highlighted by results from microelectrodes in animals (see e.g. Singer and Gray, 1995; Neuron, 1999). These single-unit recording studies in animals have been complemented by studies at coarser levels of resolution in humans and animals. These are not spikes, but local field potentials (LFP) of various degrees of spatial resolution, including scalp recordings in EEG or MEG. In fact, responses in beta $(15-30 \mathrm{~Hz})$ and gamma band $(30-70 \mathrm{~Hz})$ can be recorded during visual discrimination protocols on the human scalp (Tallon-Baudry et al., 1997) and in subdural electrocorticograms (Le Van Quyen et al., 1997a,b; Lachaux et al., 2000). Also, there is some recent evidence to suggest that not only local activities, but also long-range synchronization comparable to those found in single-unit studies in animals can also be detected between surface recordings (Rodriguez et al., 1999). In this work, it has been shown that large-scale integration implicates not only the establishment of dynamical links, but also their dismantling to give way for a next cognitive moment. The key finding here is that synchronous patterns are shortlived (lasting on the order of 50 to 250 milliseconds) with new patterns continually being created, destroyed and subsequently recreated.

These results are based on the analysis of phase synchronization, which involves the pair-wise comparison of phase relations between different electrode recordings. In this context, a classical definition of synchronization is the adjustment of the rhythmicity of two oscillators in a phaselocking:

$$
n \phi_{1}(\mathrm{t}), m \phi_{2}(\mathrm{t})=\mathrm{const}
$$

where $\phi_{1}(\mathrm{t}), \phi_{2}(\mathrm{t})$ are the instantaneous phases of the oscillators, and $n, m$ are integers indicating the ratios of possible frequency locking. In what follows we assume $n=m=1$ for simplicity, and assume that the constancy of phase difference is valid within a limited window $T$, typically of a few hundreds of milliseconds.

In the case of neural signals, detecting phase locking between two distant brain recordings (such as EEG, MEG, and intracranial) is, however, not straightforward and this quantification requires methods that are entirely different than the cross-correlograms between spike discharges of microelectrode studies. Furthermore, especially for non-invasive signals, the true synchronies are buried in a considerable background noise, which can 
give rise to spurious phase-locking between their signals. Consequently, the question "synchronous or not synchronous" can only be treated in a statistical sense (Le Van Quyen et al., 2001a). Two methods have been used to date in neuroscience, based on comparable approaches which allow a statistical estimation of the phase synchrony (Tass et al., 1998; Lachaux et al., 1999). A few steps are followed (Fig. 3A). First, the signals were filtered with a bandpass corresponding to a particular frequency component. Second, the instantaneous phase $\phi_{1}$ and $\phi_{2}$ of each filtered signal was obtained by means of the Hilbert transform (Tass et al., 1998) or a wavelet convolution

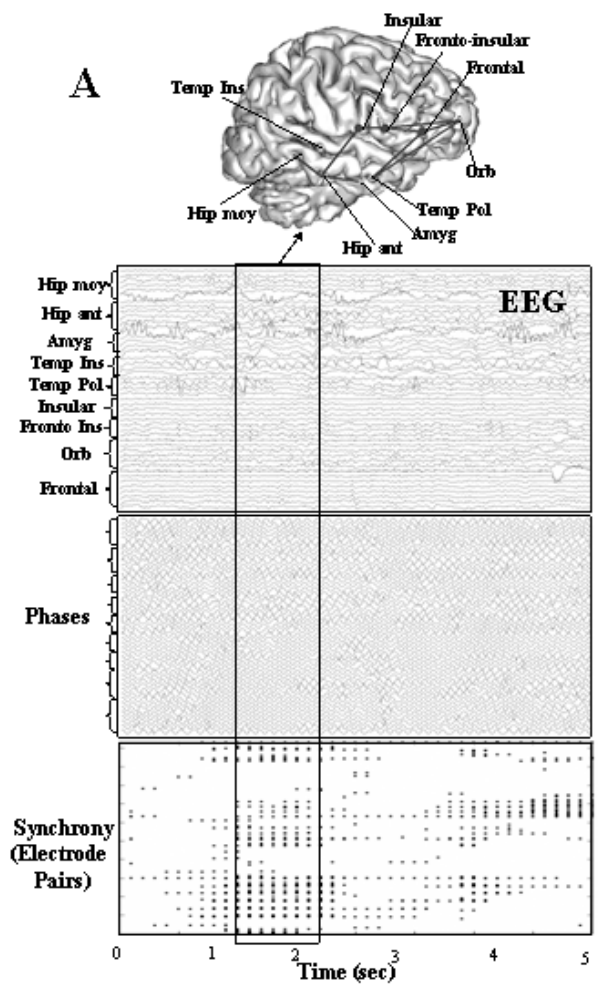

(Lachaux et al., 1999). Third, the degree of phase-locking was quantified by a statistical measure. In our work, we commonly use the variance of the phase difference onto the unit circle in the complex plane (Lachaux et al., 1999):

$$
P L S=\left\|\frac{1}{N} \sum_{t=1}^{N} e^{i\left[\phi_{1}(t)-\phi_{2}(t)\right]}\right\|
$$

where $N$ is the number of trials. This phaselocking statistics (PLS) varies between 0 (independent signals) and 1 (constant phase-lag between the two signals). The significance of each PLS is estimated via a comparison with a distribution of PLS obtained with random signals.

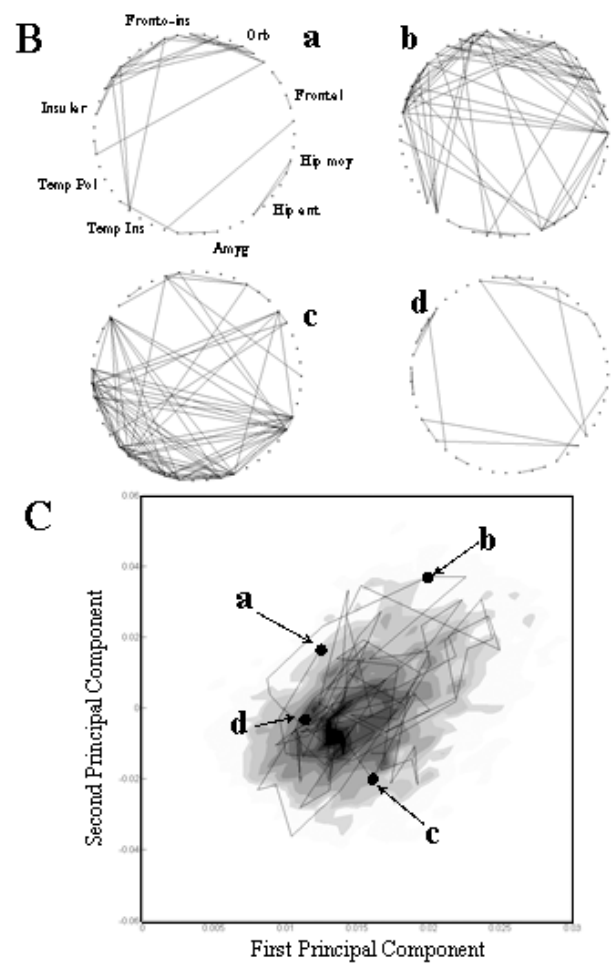

Figure 3: A: Detection of the transient synchronous patterns between intra-cranial electrodes. The key for the study of phase-synchronous activity is the pairwise comparison of the phase relations between different recording sites. Synchronization between two sites is the phase-locking of their rhythmicity, that is, their phase differences remain stable during the window of observation. In order to detect these phase adjustment, the instantaneous phase of each intracranial signal is first extracted (the second panel from the top). The degree of phase-locking is then pairwise quantified by a statistical measure of phase-locking (third panel from the top). On the top of the figure, spatial projection of synchronous patterns on a 3D MRI reconstruction for a representative window.

B: Example of transient synchronous patterns between intra-cranial electrodes shown through successive windows. We can observe that these phase-locked patterns of activity appear highly variable both temporally and spatially, transiently linking different cortical regions.

C: As a first approximation, the first two principal components of a singular value decomposition (SVD) of the graph space are here presented. The point densities are coded in a gray scale and a small part of the trajectory is shown. 
Figure 3 shows an example of synchrony characterization between intracranial EEG recordings. These data were recorded in the course of presurgical evaluation of epileptic patients who are not responsive to medication and become candidates for surgery. Far from any seizures, they provide episodes of normal, alert EEG. In contrast to scalp recordings, human intracranial EEGs are generally highly differentiated and local in respect to measures of cooperative interactions, at least down to millimeter dimensions, and fluctuate in time within seconds. Figure $3 \mathrm{~A}$ shows ongoing patterns of synchronization in the alpha range (8$13 \mathrm{~Hz}$ ) linking different deep structures during an awake state. Elevated large-scale phase synchronization can transiently be observed during periods on the order of 100 msec to a few seconds. Figure $3 B$ shows four successive graphs of synchronization. We can observe that these phase-locked patterns of activity appear highly variable both temporally and spatially, sometimes linking different cortical regions separated by several centimeters. This leads us naturally to a dynamical description of these cooperative behaviors, particularly in terms of trajectories in a space of possible states. Unfortunately, an embedding in a lowdimensional euclidean space cannot be achieved for these complex graphs as a whole. Nevertheless, as a first approximation, we can examine the first two most important principal components of a singular value decomposition of the graph space, which leads to orthogonal modes and can be used to map each time sequence into a point in 2 dimensional map. This method can be useful in getting an impression of the underlying graph dynamics and can be a good starting point for modeling these complex spatio-temporal patterns. As an example, Figure $3 \mathrm{C}$ shows this space for successive graphs of synchronization occurring during a 20-minute period of an awake state of the patient. Several observations can be described. First, a center of strong density of trajectories exists, indicating strong recurrences in the graph dynamics. This suggest that during a normal state, the large-scale dynamics exhibits a relative stability. This stability may be interpreted as a homeostasis of brain collective activity and provides a definitive baseline to compare the large-scale properties under more specific conditions of cognitive or pathological activity. Second, the trajectories constantly switch within the core, specifically creating fringe structures, creating links between different sub-regions of higher density in the graph space. This observation suggests that the graphs are intrinsically unstable and display an constant stream of new patterns. Clearly, further methodological developments are of the utmost importance for the description of the intermittent nature of this activity. Here one makes use different advances in nonlinear description to develop of a view that is truly "dynamic." Our future experimental investigations will concentrate on the existence and properties either of lowdimensional (section 6) or high order statistics (section 7).

\section{MODULATION OF THE DYNAMIC CORE BY CROSS- BAND INTERACTIONS: HIGHER ORDER SPECTRA.}

The coordination of large-scale patterns is constantly subject to a modulation of multiple synchronizing and desynchronizing influences. In particular, a variety of influences such as arousal, motivation or emotional tone could modulate this constitution. The conjunction of these consistent or conflicting influences can be seen as a contextual modulation of the dynamic core constitution. Here, topdown influences from 'higher' level processing, such as expectancy or attention, can affect the 'lower' level, such as sensorimotor processing (von Stein et al., 2000; Engel et al., 2001; Lutz et al., 2002). Since multiple influences are undoubtedly in conflict, while others are mutually consistent, it seems likely that a mechanism is at work to modulate global activity patterns in a specific way. In this context, reciprocal relations over different frequency bands have been reported to be crucial, most prominently as an interplay between high and low frequencies (Bressler et al., 1993). Specifically, a large body of evidence reported that oscillations at 
different frequencies often occur simultaneously. For instance, the corticothalamic oscillatory patterns in the alpha range $(8-13 \mathrm{~Hz})$ are known to play a crucial role in setting and resetting fast cortical oscillations in beta $(15-30 \mathrm{~Hz})$ and gamma band $(30-70 \mathrm{~Hz})$ (Steriade and Amzica, 1996). A slower pacing is provided by limbic structures involving the process of memory consolidation reflected in the theta band around $7 \mathrm{~Hz}$ (O'Keefe and Burgess, 1999). In humans, a recent study (Schack et al., 2002) reported interrelation between theta $(4-7 \mathrm{~Hz})$ and fast $(20-30 \mathrm{~Hz})$ oscillations at frontal and prefrontal electrode during memory processing. Thus, phase correlations between low frequency and gamma activity may perhaps contribute to a contextual modulation of the temporal structures of both ongoing and evoked large-scale activity.

On the basis of these considerations and other evidence recently reviewed (Engel et al., 2001), these modulating influences can be assessed by the study of cross-band interactions (Varela et al., 2001). These patterns can be directly characterized in the Fourier domain. However, ordinary spectral analysis is restricted to the investigation of amplitudes of oscillations with regard to different frequencies. Consequently, it is not possible to examine the relationship between different frequencies and to give insights about the nature of the cross-band interactions. If there exists some underlying cross-band interactions, then the phases of the Fourier modes are not independent anymore. Higher order spectra provide a means for characterizing such phase interactions and can be seen as a generalization of Fourier analysis characterizing nonlinear interaction between oscillations.

The most commonly used higher order spectra to examine the phase relationship between two different frequencies is bispectrum analysis (third order statistics) (Nikias and Petropulu, 1993). A large amount of literature is devoted to the application of this technique to EEG analysis (Dumermuth et al., 1971). As an example, the bispectral analysis provides an accurate and reliable index for assessing the anesthetic depth and is implemented in a commercially available device used in the clinical practice (Rampil, 1998). More precisely, the relationship between the sinusoids at two primary frequencies, $f_{1}$ and $f_{2}$, and a modulation component at the frequency $f_{1}+f_{2}$ is here investigated. The bispectrum of a time series $x(t)$ can be computed with a FFT to generate complex spectral values $X(f)$. For each possible triplet $\left(f_{1}, f_{2}, f_{1}+f_{2}\right)$, the complex conjugate of the spectral value at the modulation frequency $X^{*}(f 1+f 2)$ is multiplied against the spectral value of the primary frequencies of the triplet:

$\mathrm{B}\left(f_{1}, f_{2}\right)=\left|\left\langle X\left(f_{1}\right) \cdot X\left(f_{2}\right) \cdot X^{*}\left(f_{1}+f_{2}\right)\right\rangle\right|$

where $*$ denotes the complex conjugate and \langle\rangle indicates averaging over several trials. Note that the bispectrum is here defined by considering a single signal $x(t)$, but it can easily be extended to study phase coupling between different variables. If at each frequency of the triplet there is a large spectral amplitude (i.e. a sinusoid exists for that frequency) and if the phase angles for each are aligned, then the resulting product will be large. This indicates that two oscillations interact in a nonlinear way and generate a third energy component with a frequency equal to the sum of two source frequencies, i.e. the presence of quadratic phase coupling. If one of the component sinusoids is small or absent or if the phase angles are not aligned, the product will be small. By definition, the bispectrum depends on the magnitude of the signal. To exclude the influence of the magnitude and detecting solely the phase relationships, the bispectrum can be normalized by each spectral values, giving the so-called bicoherence.

Figure 4 gives representative examples of bicoherence for artificial data and intracranial EEG. For the latter, Figure 4C plots successive values of bicoherence for several deep channels, recording the amygdala and hippocampal, frontal, orbitofrontal regions during an awake state (same patient as in Fig. 3). In most of the samples, many of the bicoherence computed show virtually no significant values. In these case, a phase coupling is not typically 

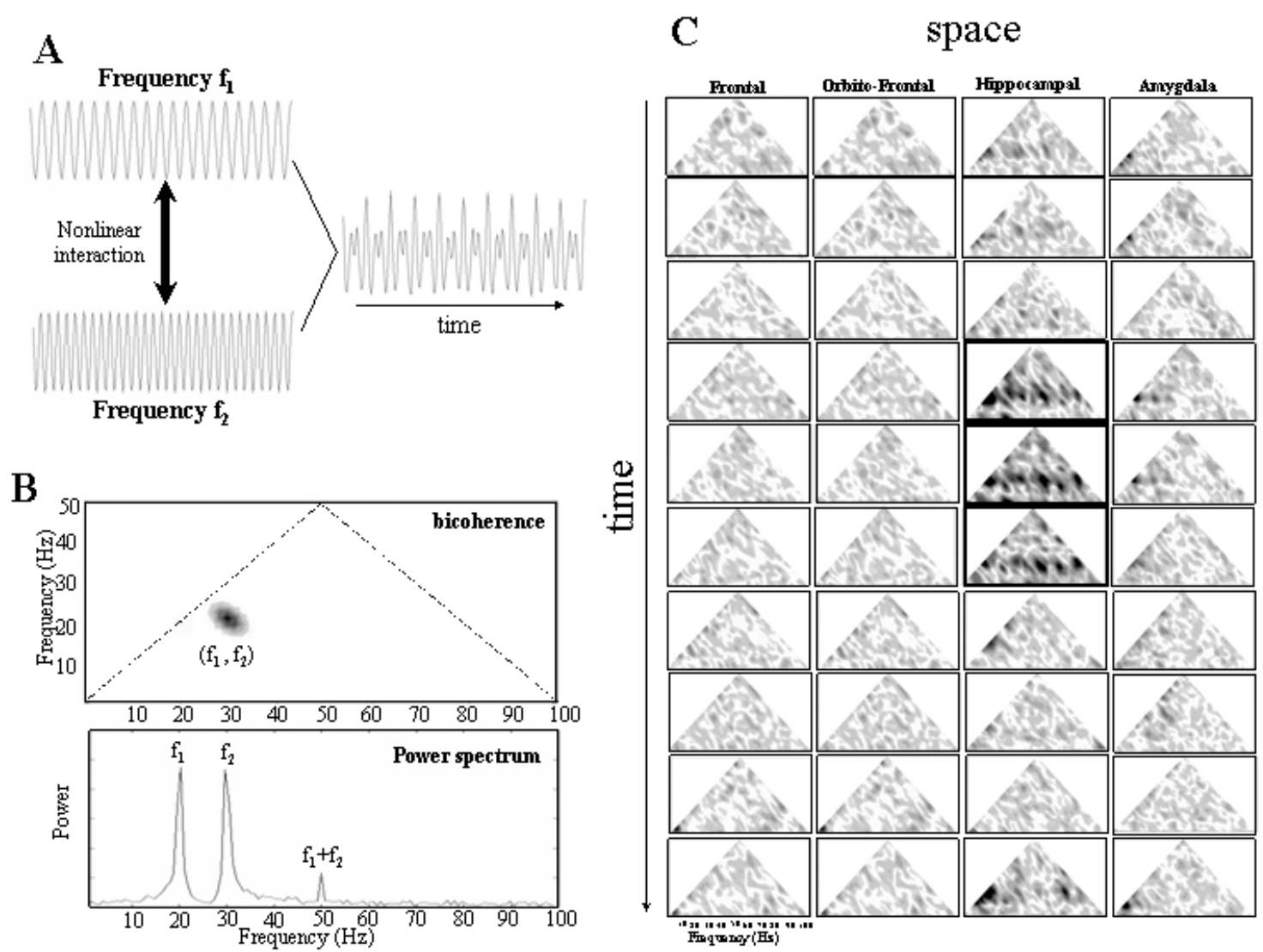

Figure 4. A: The bispectral analysis examines the nonlinear relationship between the phases of two different frequencies. B: The bicoherence is calculated in a two-dimensional space of frequency versus frequency. An artificial signal was simulated by $\mathrm{X}(\mathrm{t})=\mathrm{X}_{1}(\mathrm{t})+\mathrm{X}_{2}(\mathrm{t})+0 \cdot 2 \mathrm{X}_{1}(\mathrm{t}) \cdot \mathrm{X}_{2}(\mathrm{t})$ where $\mathrm{X}_{1}(\mathrm{t})=\exp \left[\mathrm{j}\left(2 \pi \mathrm{f}_{1}+\phi\right)\right]$ and $X_{2}(t)=\exp \left[j\left(2 \pi f_{2}+\phi\right)\right]$ with $f_{1}=20 \mathrm{~Hz}$ and $f_{2}=30 \mathrm{~Hz}$ (the upper panel shows the data). The bicoherence reveals a point of high value at $\left(f_{1}, f_{2}\right)=(20 \mathrm{~Hz}, 30 \mathrm{~Hz})$, indicating that the three frequencies $f_{1}=20 \mathrm{~Hz}, f_{2}=30 \mathrm{~Hz}$ and $f_{1}+f_{2}=50 \mathrm{~Hz}$ are in a quadratic phase coupling. The power spectrum is shown below the bicoherence. $\mathbf{C}$ : Plots successive values of bicoherence for several intra-cranial recordings. These signals were analyzed in 2048-point epochs $(5.12$ s), using a Hanning window, overlapped $50 \%$ by a sliding window. The significant bicoherences correspond to the black. As highlighted, significant phase couplings can only be identified for the hippocampal recordings between $f_{1}=15 \mathrm{~Hz}$ and $f_{2}=20,40,60,70$ and $80 \mathrm{~Hz}$.

present. Nevertheless, episodically high bicoherence values can sometimes be observed, as highlighted in Figure 4C. In our example, significant phase couplings $(p=0.05)$ were only be identified in the hippocampus between $f_{1}=15 \mathrm{~Hz}$ and higher frequencies $f_{2}=20,40,60,70$ and $80 \mathrm{~Hz}$. Note that these phase correlations were not among the harmonics (double or triple the fundamental frequency). In the case of phase correlation among harmonics, one cannot decide whether they were generated by a single or separate rhythm-generating structures. Phase correlations at nonharmonic frequencies directly indicate a quadratic phase coupling among different rhythm generating processes. In our example, these observations seem provide evidence that synchronized activity in the hippocampus on a low temporal scale (i.e. alpha) might modulate the local activities in the gamma frequency range. Furthermore, a nonlinear cooperativity was present among oscillations at different time scales. Pre-synaptic modulations could be involved in these interactions, as was recently observed in the somatosensory cortex (Haueisen et al., 2001). The limited number of samples examined to date mainly emphasize great variability within a given structure and state. Future studies must examine in detail these nonlinear 
cooperative interactions in relation to contextual modulations of both ongoing and evoked large-scale activity.

\section{EXPLORING THE UNFOLDING OF THE DYNAMIC CORE: PHASE SPACE TECHNIQUES.}

In the last decade, it has been suggested that one difficulty in analyzing complex signals was due to examining the time series in terms of a static rather than a dynamic behavior. Indeed, traditional signalprocessing procedures decompose the temporal or frequency components of the signal, for example through Fourier analysis, and thus reflect a limited amount of information (one-dimensional). In contrast, the dynamical view suggests that a time series may be seen to reflect the marks of all other hidden variables participating in the dynamics of the system (multi-dimensional). In spite of the difficulties that arise in the estimation or interpretation of phase space for neuronal signals, this approach remains simple and has profoundly modified the manner in which dynamical processes are viewed and described (Skarda and Freeman, 1987; Elbert et al., 1994; McKenna et al., 1994).

\subsection{Phase space reconstruction}

Given that a complex dynamic system can involve an enormous number of interrelated dependent variables that are impossible to measure directly, the main problem is how to analyze a multi-dimensional dynamic system knowing only a few variables that can be measured. In fact, it has been mathematically established that if we can measure any single variable with sufficient accuracy over a sufficiently long period of time, then it is possible to make quantitatively meaningful inferences about the underlying dynamical structure of the system from the behavior of that single variable (Taken, 1981). This is particularly useful with experimental neuronal activities where it is much easier to obtain a measure of a single variable at several successive times, but may be difficult to directly measure other system parameters that reflect independent degrees of freedom.

A well-established implementation of this important mathematical fact for the reconstruction of the dynamical structure from one observable is through the "method of delays" (Fig. 5-A). A "phase space" can be constructed by taking successive points of the original time series separated by a fixed time delay $\tau$. Let $V(t)$ be the neuronal activity recorded at time $t$ and let $m$ delayed amplitudes be recorded on an individual channel. At each time $t$, the state of the neuronal activity is represented as a point $A_{t}$ in a $m$ dimensional space:

$$
A_{t=}[V(\mathrm{t}), V(\mathrm{t}-\tau), \ldots, V(\mathrm{t}-(\mathrm{m}-1) \tau)]
$$

This mathematical representation permits the introduction of independent coordinates as the dynamic variables needed to specify the instantaneous state of the system. A sequence of such states followed in time defines a trajectory. An "attractor" is a trajectory in phase space to which the system will converge from any set of initial conditions.

The geometrical properties of the trajectories evolving in the phase-space can then be expressed quantitatively using nonlinear measures. An important measure is the dimensionality of the dynamics, referred to as the "correlation dimension" or $\mathrm{D}_{2}$, which characterizes the minimum number of variables that must be considered in the description. A periodic oscillation would have a dimension of 1 . A quasiperiodic oscillation (2 incommensurate frequencies) would result in a dimension of 2 . If the system has a dimension larger than 2 and is not an integer, then we would expect the system to exhibit "chaotic dynamics" that is governed by low-dimensional deterministic models and show a exponential divergence of originally nearby trajectories. Thus, any volume in phase space will tend to contract along certain directions and expand along other. There is considerable discussion concerning the parameters that would constitute sufficient accuracy for the characterization of the chaotic dynamics, how often the signal should be sampled, as well as how long data segments should be 

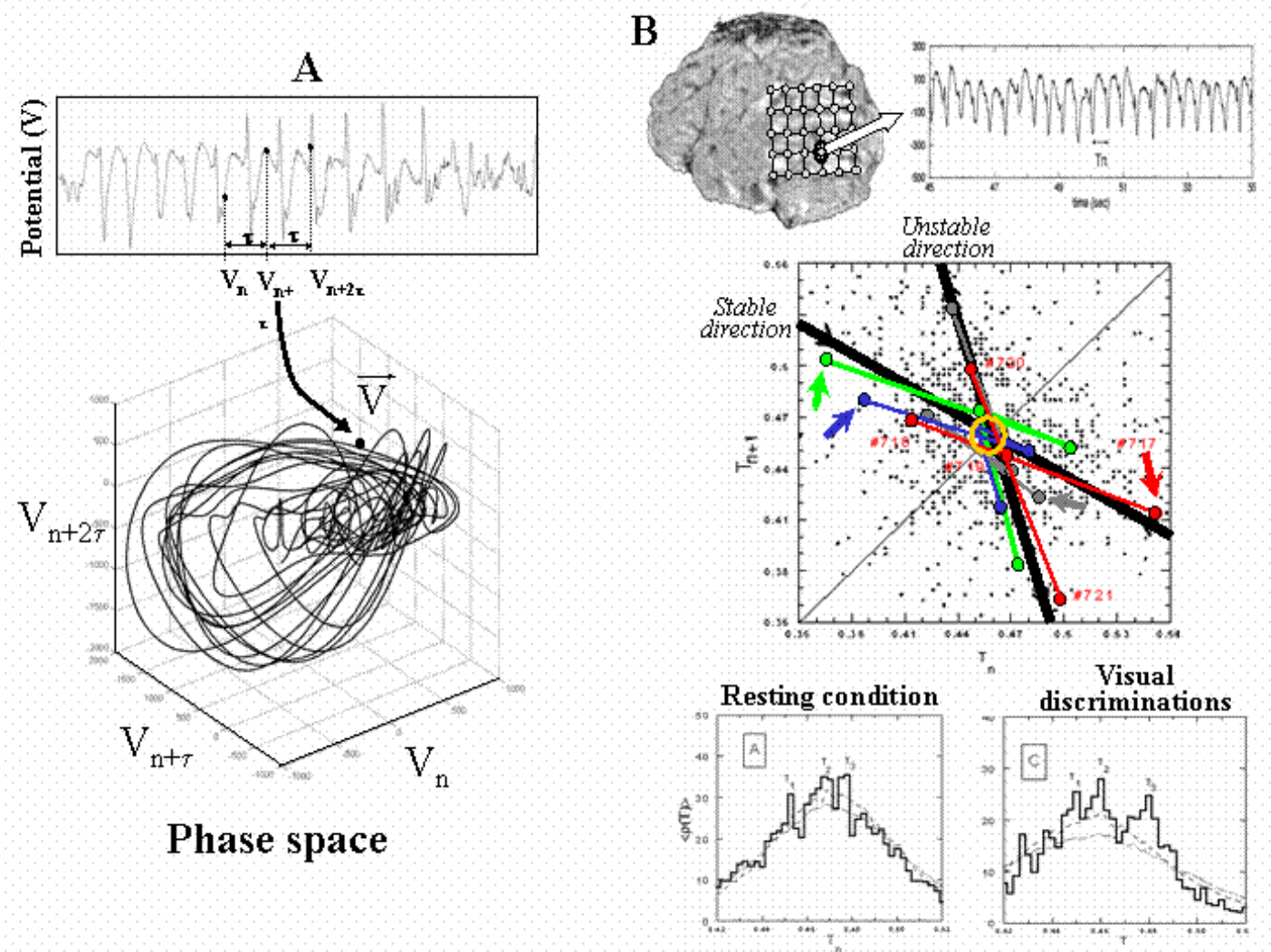

Figure 5. A: Phase-space representation: The method of delays is one way of representing the EEG dynamics in a phase space. In this representation, the state at each point in time is represented by a vector generated by taking successive amplitudes separated by a time lag $\tau$. This reconstruction of the underlying dynamics is the first step of all techniques of phase space analysis. The geometrical properties of the trajectories in the phase space can then be expressed quantitatively using nonlinear measures. B: An example of phase space reconstruction of a local EEG activity recorded from an epileptic focus. The temporal intervals between successive discharges were analyzed in a first-return map. Various clusters of periodic activities are observed (see histograms below) with recurrent trajectories toward them (stable direction) and diverging away (unstable direction).

(see Grassberger et al., 1991 for a critical review).

\subsection{An example of dynamical landscape}

Figure 5B shows an example of phase space reconstruction of a local EEG activity recorded from an epileptic focus (Le Van Quyen et al., 1997a, 1997b). Characteristic for this epileptogenic tissue, the recording exhibited spontaneous and nearly periodic interictal spikes, reflecting the manifestation of pathological recruitment of large neuronal populations, which did not produce clinical symptoms. The patient showed no evidence of cognitive impairment and was willing to participate in simple cognitive testing consisting of a visual and auditory discrimination. For the visual task, the subject was asked to press a button when the target stimulus (a reversed Kanizsa triangle) appeared, in contrast to two other stimuli (a Kanizsa triangle and a real triangle). The temporal intervals between successive discharges were analyzed in the phase-space (here a first-return map).

The analysis revealed various clusters of unstable rhythms having an underlying shorttime causality: a tendency in successive time steps to follow recurrent trajectories that approach the clusters along a specific 
direction and remain nearby before they diverge away along another unstable direction. Although the positions of the periodic points are shifted between different behavioral conditions, the related slope of approach to the instabilities appear as invariant features of the dynamics for all experimental conditions. This kind of dynamics is very similar to a chaotic trajectory which typically includes an infinite number of unstable periodic orbits. Thus the state never remains long in any of these unstable motions but continually switches from one periodic motion to another, thereby giving an appearance of randomness (So et al., 1998).

Another interesting observation is that the distance between the clusters of unstable rhythms co-varied with the specific mental state the subject was undergoing, as can be seen from a histogram of their occurrence in each case. Here, the shift of rhythms along the diagonal is approximately $20 \mathrm{msec}$, which means that the modulation is carried by activities in the gamma frequency range $(30-70 \mathrm{~Hz})$. All of this suggests that the local activity under study is transiently modulated by a gamma activity associated with the subject's cognitive states. This modulation can be accomplished because the epileptogenic zone is embedded in a complex network of other brain regions that actively participate in normal large-scale interactions. Therefore the act of perception contributes, via the phase synchrony of its associated neuronal assembly, to pull the epileptic activities towards particular unstable periodic orbits. From these observations, it follows that the global pattern of integration can produce "downward" effects (global-to-local), acting eventually on the local level of cellular assemblies (Thompson and Varela, 2001). These modulations open possibilities for a 'cognitive' control of epileptic seizures (Le Van Quyen et al., 2001b).

\subsection{Low-dimensional dynamics of neuronal ensemble activity}

The phase-space techniques have prompted an intensive search for low-dimensional deterministic phenomena in neurosciences. A first and important attempt is provided by Freeman's work (1975) which analyzed the olfactory bulb of rabbits. Based on many experimental observations, Freeman and collaborators developed a mathematical model of the EEG signals based on nonlinear coupled differential equations (Freeman et al., 1987). This model exhibits most of the important dynamical features observed in the EEG, including transitions to states reminiscent of epileptic seizures. Four types of activity can be generated by the model that are the same types actually seen in the olfactory bulb. The first type of common activity corresponds to a silent state, which seems to occur under deep anesthesia and is represented by a fixed point attractor in a phase-space. A second type is that of normal background EEG observed in the absence of a significant input. In this resting stage, the irregular fluctuation of neuronal activity was associated with a broad band power $1 /$ f spectra, very similar to chaotic dynamics. The dimensionality was calculated for the times series sampled from both the model and the empirical data and similar values were obtained. A third type of activity seen in the biological data is the reaction of the system to a learned odor. In this case, the surface EEG has an almost coherent series of inspiratory bursts of oscillations with narrow spectral distributions that vanish during each expiration. This dynamics was thought to be represented by a "limit cycle attractor." The fourth type of modeled biological activity is that of an epileptic seizure. The seizure results in a paroxysmal discharge that is induced biologically by an intense stimulation of the lateral olfactory tract. It was interpreted that the seizure results from a disconnected state in which a collapse of transmission occurs between the bulb and other parts of the system. In the model, a seizure can be simulated by changing a parameter in the excitatory feedback pathway. Freeman's work shows that the family of attractors in phase space can characterize a range of behaviors and its associated neuronal activity. Transitions between attractors and transit within attractors may be an useful descriptor for 
analyzing state changes. Another advantage of describing neuronal activity in terms of dynamical trajectories is that the same descriptors can be used at different levels of organization of the nervous systems (McKenna et al., 1994).

Another important application of phasespace techniques appeared in the context of epilepsy. Historically, Babloyantz and Destexhe (1986) were the first to demonstrate that nonlinear analysis of EEG recordings from patients with epilepsy can provide new perspectives regarding epileptogenesis. They estimated the dimensionality and the largest Lyapunov exponent (the mean rate of divergence of initially neighboring states) of scalp EEG signals recorded during a human absence seizure. They found that the correlation dimension for seizure activity is of considerably lower value than for normal activity, while a positive value was estimated for the largest Lyapunov exponent. These findings support the hypothesis that the generation of ictal activity in the brain corresponds with a specific dynamical state, which is different from normal ongoing activity. The different values of the nonlinear quantities suggest a decreased level of complexity in neuronal networks involved in the epileptic process. In a comparable study, Pijn et al. (1991) analyzed epileptic seizures in a wellcontrolled experimental animal model of epilepsy (limbic kindling in the rat). Using implanted electrodes, they analyzed the seizures over the course of time and compared the same brain area under different behavioral conditions (e.g. restful waking, exploratory locomotion and seizure). The results showed that as different brain areas were recruited into the seizure activity, there was a corresponding switch in the correlation dimension of the EEG from a high to a low one. The drop in the value of $\mathrm{D}_{2}$ was most pronounced in the primary epileptogenic area and gradually decreased with increasing distance from the focal area, suggesting a reliable relationship between location of neuronal complexity and seizure outcome. These findings are in line with the work of Iasemidis and Sackellares
(1990) who described similar variations in the largest Lyapunov exponent. Perhaps the most exciting observation, first reported by these authors, was that spatio-temporal variations of this nonlinear measure precede the seizure by several minutes. How the phase transition from interictal state to the ictal state occurs has recently been systematically explored by our group (Martinerie et al., 1998). We estimated the correlation integral (which is a measure of the average density in phase space and the fundamental statistic used to determine $\mathrm{D}_{2}$ ) from intracranial recordings hours before the seizure onsets of patients with temporal epilepsy. We demonstrated that in most cases $(90 \%)$, changes toward long-lasting states occurred before the seizure (between 2-6 minutes) and were more pronounced compared to maximal changes occurring during interictal states, thus enabling us to define a "preictal state". This phenomenon could not be detected by visual inspection of the original signal or by other more traditional methods of signal processing such as Fourier analysis. Lehnertz and Elger (1998) confirmed these findings in a comparable group of patients. Taken together, this converging evidence suggests that the dynamical properties of the preictal, ictal and postictal states are clearly different. The seizure might be interpreted as the end of a process of changes that starts long before. This transition or route tow ard the seizure seems to reflect a process from "disorder to order" and argues that epilepsy belongs to the "dynamical diseases" (Mackey and Glass, 1977).

Nevertheless, despite a great potential for the detection of subtle changes in brain dynamics, it is now known that the phase space techniques must be used very cautiously. Numerous articles have been devoted to pointing to difficulties in the practical applications of the current methods. It has been shown, for example, that finite values of the correlation dimension can be found even for "colored" random noise (i.e. for high-dimensional processes characterized by spectra with power-law dependence and random uniformly distributed phases that are clearly not related to any deterministic and low 
dimensional dynamics) (Osborne et al., 1989). Furthermore, in systems in which the exact value of the correlation dimension is theoretically known, application of a low-pass filter and a time series of finite length could lead to severe underestimation or overestimation of the dimension. In addition, if the true dimension is 5 or higher, the most commonly utilized algorithms for studying dynamical behavior produce erroneous results, sometimes spuriously suggestive of low-dimensional dynamics (Theiler et al., 1996; Hernandez et al., 1996; Lachaux et al., 1997). Consequently, measures of system complexity do not, in themselves, give any insight into whether the system under study shows a complexity change in terms of the number of degrees of freedom. Some authors have addressed this issue statistically by comparing their estimates of biological data with those of surrogate data with the same power spectrum as the original data but losing all information in the phase (Pijn et al., 1991; Martinerie et al., 1998).

7. EXPLORING THE LONG-TERM EVOLUTION OF THE DYNAMIC CORE: NON-GAUSSIAN STATISTICS

As shown by the bicoherence analysis (Section 5), many departures from Gaussianity can occur in brain dynamics. When a system is driven away from the Gaussian behavior, then high order statistics are often more appropriate than spectral or phase space representations. A promising framework for conceptualizing apparently extremely stochastic systems is given by some recent application of statistical physics. A probabilistic description is at the heart of this field, and a basic quantity that can provide significant clues into the nature of the underlying physics is the socalled probability distribution function. In this context, most of the literature deals with random variables whose probability distributions are Gaussian. Nevertheless, many complex systems manifest a probability density function that is not Gaussian, and it is $P(x)=A x^{-\mathrm{a}}$

precisely the deviation from Gaussianity that contains pertinent information about the underlying physics. The tails of the distribution, which represent large but rare events, are often of particular interest. Here, power law distributions have received much attention:

$$
P(x)=A \lambda^{-\mathrm{a}}
$$

When plotted on a log-log scale, this type of function produces a straight line of slope $-\alpha$. This power law distribution is interesting because it is scale-invariant or scale-free: When we replace the variable $x$ with a new variable $y=\lambda x$, then the distribution remains unchanged under the transformation:

$$
P(y)=\left(A \lambda^{-a}\right) P(x) \alpha P(x)
$$

We can 'zoom in' or 'zoom out' on the function while its general shape remains the same and is therefore scale invariant. These power law distributions are particularly relevant in the context of phase transition in physics. In the early 1970s, research demonstrated that many physical systems near a phase transition obeyed a series of power laws with various exponents called critical exponents (Stanley, 1971). In this state, the system does not exhibit any characteristic length scale, i.e. temporal or spatial patterns happen at all scales, unlike statistical spread around a defined mean value of a Gaussian distribution. The property of interest here is the interplay between scales, rather than the specific role played by one characteristic scale. In particular, these scale-free states reflect a tendency to be inhomegeneous in space and time, to be extremely sensitive to small microscopic perturbation and to develop "long-range" correlations over the whole system. More recently, evidence has indicated that this critical state can spontaneously self-organize in nonequilibrium systems without the necessity of fine tuning any external parameter (Bak et al., 1987). The dynamics of most of the so-called self-organized critical models (as in the original sandpile model of Bak et al., 1987) is an avalanche dynamics due, in many cases, to a threshold dynamics at the microscopic level. 
It was naturally postulated that the brain as a dynamical system is operating near critical state (Turing, 1950), and indeed, suggestive numerical simulations have been recently reported (Usher and Stemmler, 1995). Furthermore, a few signatures of criticality have already been observed in the central nervous system. For instance, cultured networks of glial cells have demonstrated a power-law relationship in the cluster-size of calcium waves (Jung et al., 1998). In another study, long-range temporal correlations have been reported for oscillation in the alpha-frequency range of spontaneous EEG-MEG recordings (Linkenkaer et al., 2001). Finally, a study in cognitive psychology showed that the time series of the errors made in reproducing time intervals has a $1 / \mathrm{f}$ power spectrum (Gilden et al., 1995).

To illustrate these considerations, we consider first a few simulated signals (Fig.6-A): white noise, 1/f noise and Brownian noise. As is well-known, white noise represents a random superposition of uncorrelated frequencies: the value at some time $t$ is totally independent of its value at any other instant. Brownian noise can be generated by a random walk as follows: the value at some time $t+1$ is obtained by adding to its previous position at time $t$ a random number (usually drawn from a Gaussian
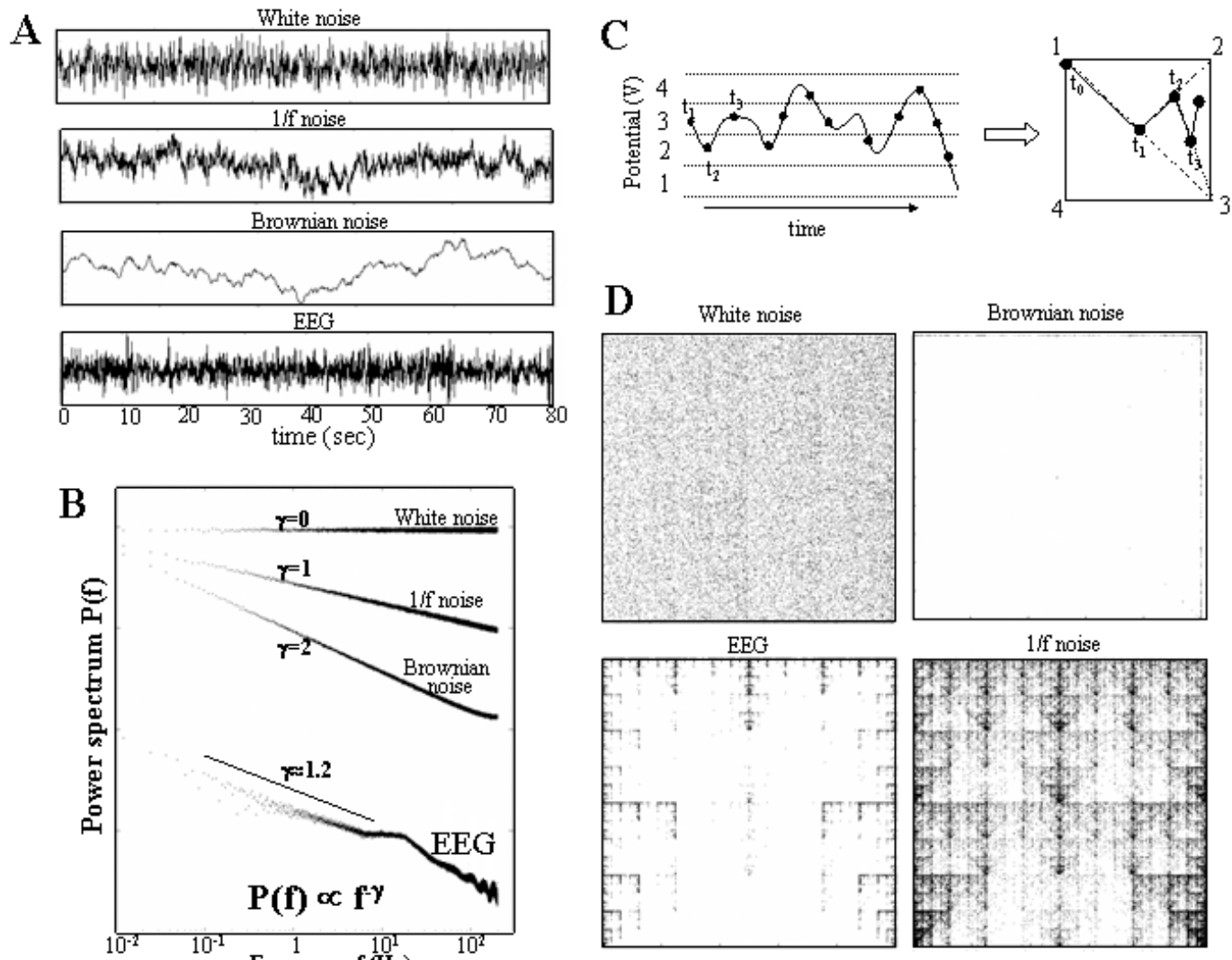

Figure 6: Long-term correlation of EEG signals.

A: Three examples of simulated signals (white noise, 1/f noise and Brownian noise) and an intra-cranial EEG. B: Power spectra of the simulated signals and an intra-cranial EEG. The power spectrum can be expressed as $\mathrm{P}(\mathrm{f}) \propto \mathrm{f}^{-\gamma}$. For $\gamma>0$, this type of power laws exhibit long trends which can be interpreted as the presence of long term correlation. Here, the FFT is averaged over 100 intervals of $82 \mathrm{sec}\left(2^{15}\right.$ points). C: The iterated function system associates to a time series a two-dimensional trajectory which gives a visual indication of the temporal structures in the signal. D: Trajectory associated with a intra-cranial recordings of an epileptic patient recorded during an awake state of 2 hours. The pattern exhibits a complex fractal structure which is clearly different than the trajectories associated with white noise or Brownian noise and similar than an artificially generated $1 /$ f noise. 
distribution). Brownian noise is therefore strongly correlated in time as the value remembers where it was a few steps ago. The $1 /$ f noise is different from the first two but shares some of their characteristics. It has a tendency toward large variations like Brownian motion, but also exhibits high frequencies like white noise. It is this type of signals which will interest us because it exhibit long trends which can be interpreted as the presence of long-term memory.

Scale invariance can be expressed simply in the Fourier domain. Usually in spectral analysis, the emphasis is placed on individual peaks which indicate particular characteristic frequencies (see Section 5). Nevertheless, the power spectrum can also be used to study relationships across time scales. As an example, Figure 6B shows the power spectrum of three simulated signals and one experimental EEG signal. As fitted on a log-log plot, the power spectrum can be expressed as $\mathrm{P}(\mathrm{f}) \propto \mathrm{f}^{-\gamma}$ with $\gamma \approx 0$ for the white noise, $\gamma=2$ for Brownian noise and $\gamma=1$ for $1 / \mathrm{f}$ noise in accordance with the theoretical values. For the latter two, P(f) diverge as f goes to zero, which favors the presence of very low frequencies and indicates long-range correlations in the signal. The important point here is that these scaling properties can also be observed in EEG activity. The data used here are from the frontal cortex of an epileptic patient recorded during an awake state of 2 hours, far away from any seizures. The power spectrum shows a slope of scaling exponents approximately $\gamma \approx 1.2$ over a range from 0.1 to $10 \mathrm{~Hz}$, in accordance with other studies (Novikov et al., 1997; Freeman et al., 2000). This means that the brain dynamics in an awake state have $1 / \mathrm{f}$ noise-like characteristics for low frequency bands.

Similar findings appeared in other patients and electrode implantations. In a nutshell, our main observations showed that first, the scaling properties seem to be time-invariant over at least a few hours, unlike spectral density that typically varies within subjects across recording conditions. Second, the power spectrum follows $\mathrm{P}(\mathrm{f}) \propto \mathrm{f}^{-\gamma}$ with $1<\gamma<2$ and can be interpreted as a "compromise" between the complete unpredictability of white noise and the very smooth landscape of Brownian noise. It would be interesting to observe in future studies whether cognitive processing, for example, resulted in a modulation of these scaling exponents.

In the view of a dynamical description, an embedding of the temporal structures in a low-dimensional space can be simply achieved using a procedure called iterated function system (Barnsley, 1988). This method associates a two-dimensional trajectory with a time series that gives a visual indication of the temporal structures in the signal. This follows several successive steps (Fig. 6C): The signal's amplitude is first classified into four bins. The amplitude bins are selected so that each receives an equal number of signal points. The original signal is then classified into the amplitude bins (labeled 1,2,3,4 in Fig. 6C). The coarse grained signal is then projected in a square where the four corners are also labeled. Each value of the coarsegrained series is associated with the corners having the same number: A point is plotted half the way between an initial point (we chose a particular corner) and the first point of the series. A second point is plotted half way between the first plotted point and the second point in the data, and so on. Therefore each point represents the entire history of a sequence leading to it. The resulting cloud of dots would converge on geometrical patterns, exhibiting hidden temporal structures of the real sequence (Miramontes and Rohani, 1998). Figure 6D shows the trajectory associated with a 2hour intracranial recording of the same patient as before. Clearly, the trajectory follows a complex structure which repeats itself at several scales and, in fact, looks like a fractal object typical of a $1 / f$ dynamics. The trajectories associated with white and Brownian noise are dramatically different. White noise fills the unit square with dots without exhibiting any particular pattern. On the other hand, Brownian noise yields a trajectory that spends most of its time near the edges of the square, due to the slow excursions of the signal.

The physiological significance of this scale-invariant behavior in brain dynamics can be manifold and raise important new 
questions. First, this observation suggests that the brain as a dynamical system operates near a critical state at a phase transition point (Stanley, 1971). This suggests that neuronal fluctuation contains structured information and therefore has an important role in cortical function (Arieli et al., 1996; Tsodyks et al., 1999). In this state, the spatiotemporal patterns remain dynamically unstable on a wide range of spatial and time scale and therefore are able to respond to very small perturbation by abrupt global reorganization (Freeman and Barrie, 1994). Second, large events occur as a consequence of the same dynamics that produces small events. In this sense, this description provides a unifying mechanism of how 'microscopic' and short-range interactions are related to 'macroscopic' large-scale phenomena. Finally, the brain is constantly creating predictions as required by continuous engagement in real-word sensorimotor contexts. Mediated by long-term temporal correlation, such predictions might be embodied in the temporal structure of ongoing activity (Engel et al., 2001). Thus, a critical state may be the dynamic memory of the neuronal networks that constrain perception and behavior to power-law statistical patterns.

\section{LOOKING TO THE FUTURE: THE INTERPLAY BETWEEN TIME AND SPATIAL SCALES}

From the above examples it becomes apparent that the property of interest is the interplay between scales, rather than the specific role played by one characteristic scale or "levels" of organization. Following Simon (1973), it is important to consider this interplay as a "loose vertical coupling," allowing the distinction between levels, and "loose horizontal coupling," allowing the separation between sub-systems at each level. While the word "loose" suggests "decomposable," the word "coupling" implies resistance to decomposition. In my view, the characterization of these "loose couplings" represents one of the essential challenges for future development.
Accordingly, future studies must examine the process of translating or extrapolating information from a fine to coarse scale, referred to as upward causation, and the process in the reverse direction or downward causation (Fig. 7). As best seen in epilepsy, a local neuronal activity at the level of an epileptogenic zone can produce upward effects, sometimes acting during the epileptic seizure on the global level and affecting the consciousness. The converse also seems to be the case, although less documented. A few case studies reported that global patterns of integration (the result of upward causation) can produce downward effects, occasionally acting on the local level of cell assemblies, whose activity can thus be taken as an indicator of the downward influence (see section 6.2). Nevertheless, there is an urgent need to conduct large-scale studies for capturing these patterns and processes and to develop strategies for translating information from global to regional and local scales (Thompson and Varela, 2001).

As was introduced before, the identification of order parameters emerging from the intrinsic dynamics of micro-events and constraining the local behavior may be of great help here. The plausibility of this view can be simply demonstrated by models of collective synchronization in coupled oscillator populations (Strogatz, 2001). For example, the Kuramoto model simulates nearly identical limit-cycle oscillators connected to all the other and following the universal form (Kuramoto, 1984):

$$
\begin{aligned}
& d \theta_{i} \\
& d t
\end{aligned}=\omega_{i}+\frac{K}{N} \sum_{j=1}^{N} \sin \left(\theta_{j}-\theta_{i}\right)
$$

where $N$ denotes the number of the oscillators, $\theta_{i}$ the phase of oscillator $i$ and $\omega_{\text {I }}$ its natural frequency. The cooperative phenomenon can be easily investigated by the complex order parameter:

$$
r e^{i \psi}=\frac{1}{N} \sum_{j=1}^{N} e^{i \theta j}
$$

This macroscopic quantity can be interpreted as the collective rhythm produced by the synchronization of the entire population. For instance, if all the oscillators move in a single cluster, we 
A

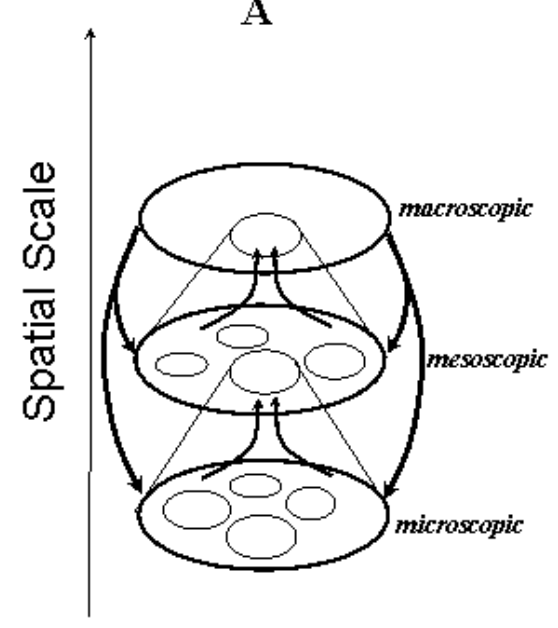

B

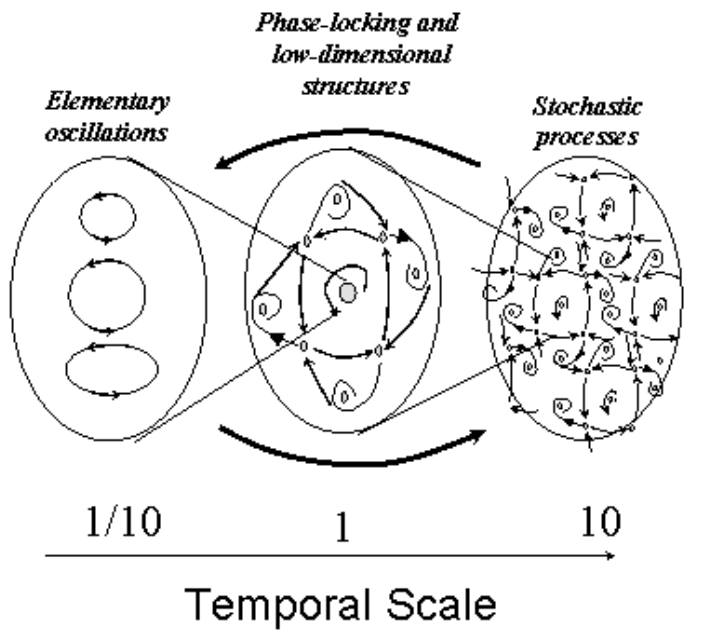

\section{Figure 7: Across time and spatial scales.}

A: Sketch illustrating the dynamical interdependence between the microscopic scale and the emerging global structures at the mesoscopic and macroscopic level. The upward arrows indicate that microscopic interactions tend to spontaneously synchronize their behavior; which initiates the beginnings of a mesocopic and macroscopic, ordered state. As indicated by the downward arrows, this newly forming state acts upon the microscopic interactions and constrains further synchronizations. Through the continuing, interplay between the scales, the emergent, self-organizing structure is stabilized and actively maintained.

B: Sketch illustrating the dynamical interdependence of brain endogenous dynamics. Three scale can be distinguished: the scale of the intrinsic cellular rhythms below $100 \mathrm{msec}(1 / 10 \mathrm{scale})$, the scale of large scale integration on the order of 100-300 msec ( 1 scale) and the scale of long-term processes over a few seconds (10 scale). Low-dimensional structures may constitute the basis of the 1 scale and the processes at the 10 scale are best described by a stochastic randomness.

have $r \approx 1$ and the population acts like a giant oscillator. To the contrary, if the phases of the oscillators are scattered, then $r \approx 0$ and no macroscopic rhythm is produced. As introduced by Kuromoto, the governing equation can be rewritten in terms of the order parameter:

$$
\frac{d \theta_{i}}{d t}=\omega_{i}+K r \sin \left(\psi-\theta_{i}\right)
$$

In this form, the circular causality between the macroscopic levels (here the order parameter of amplitude $r$ and phase $\psi$ ) and microscopic levels (the phase $\theta_{i}$ of an oscillator) becomes obvious. Specifically, each oscillator appears only to be coupled to the order parameter of amplitude $r$ and phase $\psi$ by nonlinear relation: the phase $\theta_{i}$ becomes pulled toward the mean phase $\psi$, rather than toward the phase of any individual oscillator. Moreover, the strength of the synchronization is proportional to the amplitude $r$. This proportionality sets up a positive feedback loop: as the population becomes more coherent, $r$ grows and so the coupling $\mathrm{Kr}$ increases, which tends to recruit more oscillators into the synchronized cluster. From this perspective, the nonlinear relationship between two characteristic domains of scale in space can be investigated in mathematical terms and bring the idea of downward causation closer to the neurophysiological reality.

\section{CONCLUSIONS}

In summary, this paper illustrates the increasing need to mathematically describe 
global processes in the search for better explanations of how the brain self-organizes distributed neuronal activities. Based on Francisco Varela's work, my purpose here was to sketch a rough cartography that offers a mathematical framework to analyze spatio-temporal large-scale phenomena. The techniques presented here (synchronization, higher order spectra, phase-space methods, and non-gaussian statistics) are promising for providing new information to progress into the understanding of brain nonlinear dynamics and are likely to gain wider acceptance. Specifically, I argue that these different descriptions are adapted to intrinsically different domains of scale in time and space. Furthermore, the characterization of the interplay between scales in brain dynamics, rather than the specific role played by one characteristic scale alone, will represent one of the essential challenge for future developments. Given that the global brain dynamics comprises multi-leveled or multiscaled patterns and processes, the main purpose of future research is to address the following questions: Are there general rules for scaling up or down? How do the number of scales (or hierarchical levels), nonlinearity, and patchiness affect the feasibility and accuracy of scaling? How much detail needs to be incorporated (or discarded) for a given scaling purpose? Is it possible or necessary to scale from the cell up to the large-scale behavior? More to the point, how? I believe a successful description of the large-scale brain activities must be able to effectively tackle these complex aspects. From our present point of view, there is here no doubt that the analysis of nonlinear brain processes is still open to considerable progress. What is still missing is a stronger cross-disciplinary interaction, which is essential for fostering our understanding of brain dynamic phenomena. In particular, many tools developed for nonlinear analysis are still waiting for a straightforward link with a clear interpretation of underlying neurophysiology. Future developments have to bridge this gap between neurosciences and formal descriptions.

\section{REFERENCES}

ARIELI A, STERKIN A, GRINVAL A, AERTSEN A (1996) Dynamics of ongoing activity: explanation of the large variability in evoked cortical responses. Sci 273: 1868-1871

ASHBY R (1952) Design for a Brain. London: ChapmanHall

BABloyAntZ A, DESTEXHE A (1986) Low dimensional chaos in an instance of epilepsy. Proc Natl Acad Sci USA 83: 3513-3517

BAK P, TANK C, WIESENFELD K (1987) Self-organized criticality: an explanation of 1/f noise. Phys Rev Lett 59: $381-384$

BARNSLEY M (1988) Fractal everywhere. London: Academic Press

BRESSLER SL, COPPOLA R, NAKAMURA R (1993) Episodic multiregional cortical coherence at multiple frequencies during visual task performance. Nature 366: 153-156

CHANDRASEKHAR S (1961) Hydrodynamic and Hydromagnetic stability. Oxford: Oxford University Press

DAMASIO AR (1990) Synchronous activation in multiple cortical regions: a mechanism for recall. Seminars in The Neurosciences 2: 287-296

DUMERMUTH G, HUBERT PJ, KLEINER B, GASSER T (1971) Analysis of the interrelations between frequencies bands of the EEG by means of the bispectrum: A preliminary study. Electroenceph Clin Neurophysiol 31: 136-148

ELBERT T, RAY WJ, KOWALIK AJ, SKINNER JE, GRAF KE, BIRBAUMER N (1994) Chaos and physiology: deterministic chaos in excitable cell assemblies. Physiol Rev 74: 1-47

ENGEL AK, FRIES P, SINGER W (2001) Dynamic predictions: oscillations and synchrony in top-down processing. Nature Rev Neurosci 2: 704-716

FREEMAN WJ (1975) Mass action in the nervous system. New York: Academic Press

FREEMAN WJ (1987) Stimulation of chaotic EEG patterns with a dynamic model of the olfactory system. Biol Cybern 56: 139-150

FREEMAN WJ, BARRIE JM (1994) Chaotic oscillations and the genesis of meaning in cerebral cortex. In: BUZSAKI, LLINAS R, SINGER W, BERTHOZ A, AND CHRISTEN Y G (eds) Temporal Coding in the brain. Berlin: Springer-Verlag. pp 13-37

FREEMAN WJ, ROGER LJ, HOLMES MD, SILBERGELD DL (2000) Spatial spectral analysis of human electrocorticograms including alpha and gamma bands. J Neurosci Meth 95: 111-121

FRISTON KJ (2000) The labile brain. I. Neuronal transients and non-linear coupling. Phil Trans R Soc London B 355: $215-236$

GILDEN DL, THORNTON T, MALLON MW (1995) 1/f noise in human cognition. Sci 267: 1837-1839

GRASSBERGER P, SCHREIBER T, SCHAFFRATH C (1991) Nonlinear time series analysis. Int J Bif Chaos 1: $521-547$

HAUEISEN J, SCHACK B, MEIER T, CURIO G, OKADA $Y$ (2001) Multiplicity in the high-frequency signals ensuring the short-latency somatosensory evoked cortical activity in humans. Clinical Neurophysiol 112: $1316-1325$ 
HERNANDEZ J, VALDES PA, VILA P (1996) EEG spike and wave modeled by a stochastic limit cycle. NeuroRep 7: 2246-2250

HOPFIELD JJ (1982) Neural networks and physical systems with emergent collective computational abilities. Proc Natl Acad Sci USA 79: 2554-2558

IASEMEDIS LD, SACKELLARES JC, ZAVERI HP, WILLIAMS WJ (1990) Phase space topography and the Lyapunov exponent of electrocorticograms in partial seizures. Brain Topogr 2: 187-201

JUNG P, CORNELL-BELL A, SHAVER MADDEN K, MOSS F (1998) Noise-induced spiral waves in astrocyte syncytia show evidence of self-organized criticality. J Neurophysiol. 79: 1098-1101

KELSO JS (1995) Dynamic Patterns: The self-organization of brain and behavior, Cambridge, MA: MIT Press,

KURAMOTO Y (1984) Chemical oscillations, Waves and Turbulence, Berlin: Springer

LACHAUX JP, PEZARD L, GARNERO L, PELTE C, RENAULT B, VARELA FJ, MARTINERIE J (1997) Spatial extension of brain activity fools the singlechannel reconstruction of EEG dynamics. Human Brain Mapping 5: 26-47

LACHAUX JP, RODRIGUEZ E, MARTINERIE J, VARELA FJ (1999) Measuring phase-synchrony in brain signal. Human Brain Mapping 8: 194-208

LACHAUX JP, RODRIGUEZ E, MARTINERIE J, ADAM C, HASBOUN D, VARELA FJ (2000) Gamma-band activity in human intracortical recordings triggered by cognitive tasks. Eur J Neurosci 12: 2608-2622

LEHNERTZ K, ELGER CE (1998) Can Epileptic Seizures be predicted? Evidence from Nonlinear Time Series Analysis of Brain Electrical Activity. Phys Rev Lett 80: 5019-5022

LE VAN QUYEN M, ADAM C, LACHAUX JP, MARTINERIE J, BAULAC M, RENAULT B, VARELA FJ (1997a) Temporal patterns in human epileptic activity are modulated by perceptual discriminations. Neuroreport 8: 1703-1710

LE VAN QUYEN M, MARTINERIE J, ADAM C, VARELA FJ (1997b) Unstable periodic orbits in a human epileptic activity. Physical Rev E 56: 3401-3411

LE VAN QUYEN M, FOUCHEZ J, LACHAUX JP, RODRIGUEZ E, LUTZ A, MARTINERIE J, VARELA F (2001a) Comparison of Hilbert Transform and wavelet methods for the analysis of neuronal synchrony. J Neurosci Meth 111: 83-98

LE VAN QUYEN M, MARTINERIE J, NAVARRO V, BAULAC M, VARELA FJ (2001b) Characterizing neurodynamic changes before seizures. J Clin Neurophysiol 18: 191-208

LINKENKAER K, NIKOULINE VV, PALVA JM, ILMONIEMI J (2001) Long-range temporal correlations and scaling behavior in human brain oscillations. J of Neurosci 21: 1370-1377

LUMER ED, EDELMAN GM, TONONI G (1997) Neural dynamics in a model of the thalamocortical system.1. Layers, loops and the emergence of fast synchronous rhythms. Cereb Cortex 7: 207-227

LUTZ A, LACHAUX JP, MARTINERIE J, VARELA FJ (2002) Guiding the study of brain dynamics by using first-person data: synchrony patterns correlate with ongoing conscious states during a simple visual task. Proc. Natl. Acad. Sci. USA 99: 1586-1591

MACKEY MC, GLASS L (1977) Oscillation and chaos in physiological control system, Science $197: 287-289$
MARTINERIE J, ADAM C, LE VAN QUYEN M, BAULAC M, CLÉMENCEAU S, RENAULT B, VARELA F (1998) Epileptic seizures can be anticipated by non-linear analysis. Nature Medicine 4: 1173-1176

MCKENNA TM, MCMULLEN TA, SHLESINGER MF (1994) The brain as a dynamic physical system. Neuroscience 60: 587-605

MIRAMONTES O, ROHANI P (1998) Intrinsically generated coloured noise in laboratory insect populations. Proc R Soc Lond B 265: 785-792

NEURON (1999) 24, Cell Press (special volume devoted to reviews on the binding problem)

NICOLIS G, PRIGOGINE G (1977) Self-organization in nonequilibrium systems. New-York Wiley

NIKIAS LC, PETROPULU AP (1993) Higher-Order Spectra Analysis, Englewood Cliffs, New Jersey, USA: PTR Prentice Hall,

NOVIKOV E, NOVIKOV A, SHANNAHOFF-KHALSA D, SCHWARTZ B, WRIGHT J (1997) Scale-similar activity in the brain. Phys Rev E 56: R2387-2389

OKEEFE J, BURGESS N (1999) Theta activity, virtual navigation and the human hippocampus. Trends $\mathrm{Cog}$ Sci 3: 403-406

OSBORNE AR, PROVENZALE A (1989) Finite correlation dimension for stochastic systems with power-law spectra. Physica D 35: 357-381

PEZARD L, MARTINERIE J, BRETON F, BOURZEIX JC, RENAULT B (1994) Nonlinear forecasting measurements of multichannel EEG dynamics. Electroenceph. Clinic Neurophysiol 91: 383-391

PIJN JP, NEERVEN JV, NOEST A, LOPES DA SILVA FH (1991) Chaos or noise in EEG signals: dependence on state and brain site. Electroenceph Clinic Neurophysiol 79: 371-381

RAMPIL IJ (1998) A primer for EEG signal processing in anesthesia. Anesthesiology 89: 980-1002

RODRIGUEZ E, GEORGE N, LACHAUX JP, MARTINERIE J, VARELA FJ (1999) Perception's Shadow: Long-distance synchronization in the human brain. Nature 397: 340-343

RUDRAUF D, LUTZ A, COSMELLI D, LACHAUX JP, LE VAN QUYEN M (2003) From Autopoiesis to Neurophenomenology: Francisco Varela's exploration of the biophysics of being. Boil Res 36 (this issue)

SCHACK B, VATH N, PETSCHE H, GEISSLER HG, MÖLLER E (2002) Phase-coupling of theta-gamma EEG rhythms during short-term memory processing. Intl J of Psychophysiol 44: 143-163

SCHREIBER T (1999) Interdisciplinary application of nonlinear time series methods, Phys Rep 308: 1-40

SIMON HA (1973) The organization of complex systems. In Pattee HH (ed), Hierarchy Theory: The Challenge of Complex Systems. New York: George Braziller. pp: $1-27$

SINGER W, GRAY CM (1995) Visual feature integration and the temporal correlation hypothesis. Annu Rev Neurosci 18: 555-586

SKARDA CA, FREEMAN WJ (1987) How brains make chaos in order to make sense of the word. Behav Brain Sc. 10: 161-195

SO P, FRANCIS JT, NETOFF TI, GLUCKMAN B.J., SCHIFF SJ (1998) Periodic orbits: a new language for neuronal dynamics. Biophys J 74:2776-2785

STANLEY HE (1971) Introduction to phase transition and critical phenomena. London: Oxford University Press 
STERIADE M, AMZICA F (1996) Intracortical and corticothalamic coherency of fast spontaneous oscillations. Proc Natl Acad Sci USA 93: 2533-2538

STROGATZ SH (2001) Exploring complex networks. Nature 410: 268-276

TAKEN F (1981) Lecture Notes in Mathematics: Dynamical systems and turbulence, Berlin: Springer Verlag

TALLON-BAUDRY C, BERTRAND O, DELPUECH C, PERNIER J (1997) Oscillatory gamma-band (30-70 $\mathrm{Hz}$ ) activity induced by a visual search task in human. J Neurosci 17: 722-734

TASS P, ROSENBLUM MG, WEULE J, KURTHS J, PIKOVSKY A, VOLKMANN J, SCHNITZLER A, FREUND HJ (1998) Detection of $n$ :m phase locking from noisy data: application to magnetoencephalography. Physical Rev Letters 81:3291-94

THEILER J, RAPP PE (1996) Re-examination of the evidence for low-dimensional nonlinear structure in the human electroencephalogram. Electroenceph and Clin Neurophysiol 98: 213-223

THOMPSON E, VARELA FJ (2001) Radical embodiment: neuronal dynamics and consciousness. Trends in Cognitive Science 5: 418-425

TONONI G, EDELMAN GM (1998) Consciousness and complexity. Sci 282: 1846-1851
TSODYKS M, KENET T, GRINVAL A, ARIELI A (1999) Linking spontaneous activity of single cortical neurons and the underlying functional architecture. Sci 286 : 1943-1946

TURING AM (1950) Computing machinery and intelligence. Mind 59: 433-460

USHER M, STEMMLER M (1995) Dynamic patterns leads to $1 / \mathrm{f}$ noise in neuronal populations. Phys Rev Lett 74: $326-329$

VARELA FJ (1979) Principles of biological autonomy. Amsterdam: North Holland

VARELA FJ (1995): Resonant cell assemblies: A new approach to cognitive functions and neuronal synchrony. Biol Res 28: 81-95

VARELA FJ (1999) The specious present. In: PETITOT J, VARELA FJ, PACHOUD B, ROY JM (eds) Naturalizing phenomenology. Stanford, CA: Stanford University Press

VARELA F, LACHAUX JP, RODRIGUEZ E, MARTINERIE J (2001) The brain web: phase synchronization and large-scale integration. Nature Rev Neurosci 2: 229-239

VON STEIN A, CHIANG C, KONIG P (2000) Top-down processing mediated by interareal synchronization. Proc Natl Acad Sci (USA) 97: 14748-14753

WRIGHT JJ, LILEY DTJ (1996) Dynamics of the brain at global and microscopic scales: neuronal networks and the EEG. Behav and Brain Sci 19: 285-320 